\title{
Aprendizaje socioemocional en preescolar: fundamentos, revisión de investigaciones y propuestas
}

\section{Social-Emotional Learning in Preschool: Foundations, Review of Research, and Proposals}

\author{
Carmen Yolanda Guevara Benitez (*) https://orcid.org/0000-0001-5659-7246 \\ Juan Pablo Rugerio Tapia (*) https://orcid.org/0000-0002-9187-5632 \\ Ángela María Hermosillo García (*) https://orcid.org/0000-0001-6652-6521 \\ Laura Alejandra Corona Guevara (*) https://orcid.org/0000-0002-7244-7149 \\ (*) Universidad Nacional Autónoma de México \\ (Recibido: 28 de febrero de 2019; Aceptado para su publicación: 3 de junio de 2019)
}

Cómo citar: Guevara, C. Y., Rugerio, J. P., Hermosillo, A. M. y Corona, L. A. (2020). Aprendizaje socioemocional en preescolar: fundamentos, revisión de investigaciones y propuestas. Revista Electrónica de Investigación Educativa, 22, e26, 1-14. https://doi.org/10.24320/redie.2020.22.e26.2897

\section{Resumen}

El trabajo tiene como objetivo exponer los fundamentos teóricos y metodológicos para diseñar e implementar un currículum encaminado al desarrollo de habilidades sociales y emocionales dentro de las escuelas preescolares del contexto latinoamericano. Se señalan las razones que justifican la incorporación de un programa de desarrollo socioemocional en el currículum preescolar, las bases teóricas que fundamentan la importancia de la enseñanza de esta área de desarrollo psicológico infantil, así como los hallazgos de investigaciones empíricas realizadas en el contexto hispano y latinoamericano. Se concluye con la exposición de los lineamientos para la implementación de un currículum que abarque la enseñanza de la competencia social y emocional en escuelas preescolares latinoamericanas, fundamentados a nivel teórico y metodológico.

Palabras clave: Competencias interpersonales, desarrollo emocional, preescolares, enseñanza, evaluación.

\section{Abstract}

This study seeks to present the theoretical and methodological foundations for the design and implementation of a curriculum aimed at developing social and emotional skills in preschools in a Latin American context. It lays down the reasons justifying the inclusion of a social-emotional development program in the preschool curriculum, the theoretical foundations underpinning the importance of teaching this aspect of child psychological development, and the findings of empirical studies conducted in a Hispanic and Latin American context. The work concludes by offering theoretically and methodologically grounded guidelines for the implementation of a curriculum that includes teaching social and emotional competence in Latin American preschools. 


\section{Introducción}

El presente trabajo tiene como objetivo la exposición de los lineamientos -fundamentados teórica y metodológicamente- para la implementación de un currículum que abarque la enseñanza de la competencia socioemocional en escuelas preescolares latinoamericanas. Se hace énfasis en los hallazgos de investigaciones empíricas psicológicas de los últimos 10 años, localizadas en bases electrónicas de datos (Latindex, Redalyc, DOAJ y SciELO), utilizando descriptores como: educación socioemocional, habilidades sociales, preescolares y programas de intervención.

La Organización de las Naciones Unidas para la Educación, la Ciencia y la Cultura (UNESCO, 2015) señala que la finalidad de la educación del siglo XXI es apoyar y aumentar la dignidad, la capacidad y el bienestar de las personas, y su relación con los demás y con la naturaleza. Ello implica tomar en cuenta una serie de valores al orientar los contenidos y procedimientos formativos y curriculares de cada contexto y sistema educativo.

La trascendencia de la enseñanza de habilidades sociales en centros educativos es explicada por Ríos y Marchena (2017): la convivencia escolar ha tenido un deterioro progresivo, que está relacionado con el deterioro de diversos aspectos como objetivos escolares, rendimiento académico y desarrollo socioemocional. Un indicador de ello es el creciente acoso escolar y la tolerancia hacia sus manifestaciones, con serias consecuencias para el alumnado (p. 302). Es necesario reconocer que uno de los aspectos que puede influir en ello es la implementación de una metodología competitiva en los centros escolares y la poca atención hacia la enseñanza de habilidades socioemocionales, que se traduce en problemas como baja concentración, irritabilidad, conflictos interpersonales, aislamiento social y disminución en el desempeño académico (González et al., 2018).

En ese marco, se ha desarrollado en Estados Unidos y países europeos un enfoque denominado Aprendizaje Social y Emocional (SEL, por sus siglas en inglés), cuyo objetivo es integrar, de manera estructurada, los avances científicos, métodos de instrucción y materiales disponibles para que los profesionales de la enseñanza promuevan el ajuste socioemocional y el éxito académico de los estudiantes de diversos niveles educativos (Weissberg et al., 2015). Para ello, se recurre a planteamientos teóricos psicológicos que explican los aspectos que intervienen en el desarrollo de habilidades socioemocionales y cognoscitivas. El SEL probó su efectividad a tal grado que en Estados Unidos se establecieron políticas educativas para implementar programas en 50 estados, como parte del currículum académico, en todos los niveles educativos.

Sería sencillo adoptar los programas SEL en países como México, pero debe considerarse que las escuelas estadounidenses, y los problemas que los alumnos enfrentan, pueden ser distintos a los que se dan en otro tipo de países. Por ello, el presente trabajo expone una revisión de los principales planteamientos de la psicología del desarrollo y hallazgos de investigaciones empíricas realizadas en países de habla hispana.

Según la Organización para la Cooperación y el Desarrollo Económico (OCDE, 2015), entre los principales problemas en países latinoamericanos destacan la violencia, el bajo desempeño escolar, así como los problemas emocionales y de salud mental, que se traducen en altos índices de delincuencia y suicidio. Ante este panorama, se plantea la necesidad de desarrollar habilidades socioemocionales en los niños, paralelamente con la educación tradicional, porque la construcción de relaciones puede mejorar la calidad de vida de las personas, reducir la delincuencia y mejorar el clima social.

Un programa que privilegie la prevención de tales problemas sociales debe ser implementado desde el nivel preescolar, porque los niños en esa etapa, con la guía de los adultos, inician la comprensión y regulación de sus emociones. Ello permite el desarrollo de conductas socioemocionales adecuadas, es decir, que se enmarcan en las pautas definidas y aceptadas socialmente para una cultura particular, incluyendo valores y comportamientos dirigidos al cumplimiento de los derechos de las personas, así como al cuidado del ambiente, la familia, la escuela y la comunidad (Pinto et al., 2015).

Es importante que se plantee la educación socioemocional como un objetivo educativo formal, y se diseñe un currículum para que los alumnos desarrollen habilidades cognoscitivas, emocionales y sociales que 
pueden ayudarlos en su vida cotidiana y académica. Ello requiere recurrir a los fundamentos teóricos psicológicos, así como a los hallazgos de la investigación empírica, que proporcionan una fundamentación metodológica para el diseño e implementación de tales programas.

\section{Fundamentos teóricos sobre habilidades sociales, cognoscitivas y emocionales}

La psicología del desarrollo infantil plantea que el aprendizaje de habilidades sociales se inicia desde las primeras interacciones entre un bebé y sus cuidadores. Durante los años preescolares se acelera el ritmo de aprendizaje del niño respecto de su mundo social, a través de un proceso de asimilación de normas, reglas y costumbres que corresponden a su cultura, lo cual permite que desarrollen una interpretación del mundo y un concepto sobre sí mismos (Piaget et al., 1982).

La perspectiva cognitivo-conductual sentó las bases para definir la competencia social. Caballo (1993) plantea que aprender habilidades sociales y disminuir obstáculos como la ansiedad y los pensamientos negativos son aspectos importantes en la formación del individuo. La competencia social se define, según este autor, como:

El conjunto de conductas emitidas por un individuo en un contexto interpersonal, que expresa los sentimientos, actitudes, deseos, opiniones o derechos de ese individuo de un modo adecuado a la situación, respetando esas conductas en los demás, y que generalmente resuelve los problemas inmediatos de la situación mientras minimiza la probabilidad de futuros problemas. (p. 6).

La competencia social involucra un componente afectivo-emocional que incluye apego, expresividad y autocontrol; un componente cognitivo que se relaciona con conocimiento social, asumir una perspectiva y explicación de atribuciones, así como el razonamiento moral; y un tercer componente que es propiamente la conducta social, que se refiere a habilidades de comunicación verbal y no verbal, cooperación, apoyo, participación y manejo de conflictos (Caballo, 1993).

Las conductas y estrategias involucradas en la competencia social permiten a la persona desarrollarse en distintos contextos individuales e interpersonales, que pueden cambiar de cultura a cultura. Por ello, sólo pueden ser aprendidas a través de la integración e interacción en contextos sociales (Caballo et al., 2017; Mendo et al., 2016).

Monjas (2002) señala que:

Actualmente es prácticamente unánime la opinión de que las habilidades sociales se adquieren mediante una combinación del proceso de desarrollo y del aprendizaje. Ningún niño nace simpático, tímido o socialmente hábil; a lo largo de la vida se va aprendiendo a ser de un modo determinado. (p. 31).

Al igual que otros tipos de conducta, el aprendizaje socioemocional se da a través de cuatro mecanismos: 1) por experiencia directa, en función de las consecuencias reforzantes o aversivas; 2) por observación, como resultado de la exposición a modelos significativos y simbólicos; 3) a través de interacciones lingüísticas en forma de preguntas, instrucciones, incitaciones, explicaciones o sugerencias, y 4) por retroalimentación (feedback) interpersonal, en donde cada individuo involucrado en una interacción brinda información al otro sobre su comportamiento; la retroalimentación positiva puede considerarse como un reforzamiento social, mientras que la negativa sirve de guía para realizar ajustes en el comportamiento social (Monjas, 2002).

La psicología cognitivo-conductual ha dedicado décadas de investigación al desarrollo de programas de intervención dirigidos a personas que presentan déficits en habilidades sociales y trastornos de conducta asociados. Adoptar este modelo representa una serie de ventajas, tales como: el diseño de programas sistemáticos por grados de dificultad, el uso de técnicas de enseñanza de probada eficacia, el planteamiento de objetivos de intervención y criterios de logro, así como la implementación de estrategias 
de evaluación para las diferentes áreas de desarrollo implicadas en el aprendizaje socioemocional.

La enseñanza-aprendizaje puede darse en situaciones estructuradas, con enseñanza directa y explícita, o en situaciones informales, donde la enseñanza es indirecta. Como señalan Guevara et al. (2018), la mayor parte de las prácticas de crianza infantil se realizan a través de situaciones poco estructuradas (informales); los padres no necesariamente se plantean la enseñanza socioemocional, dicho proceso se realiza a través del aprovechamiento de actividades cotidianas. Cuando el niño manifiesta una conducta suele recibir retroalimentación de los adultos (o de niños de mayor edad); por ejemplo, le advierten que una conducta no es correcta o que es peligrosa, o lo felicitan por hacer algo bueno. Muchas situaciones van acompañadas de un juicio moral sobre la conducta del niño o de otras personas, incluidos personajes de ficción vistos en televisión o en libros de cuentos.

El proceso de socialización dentro de la familia incluye el aprendizaje de reglas sociales, patrones emocionales y de conducta, así como formas de interacción. Esto se realiza a través de los estilos de crianza o patrones de interacción familiar. La tipología más conocida, que tiene como origen las investigaciones de Baumrind (1966), delimita cuatro estilos: autoritario, cuando los padres exigen obediencia, imponen reglas, no permiten el diálogo, y recurren al castigo; permisivo, cuando los padres son tolerantes en extremo; negligente, con padres que no están pendientes de las necesidades de sus hijos, y democrático o autoritativo, cuando las familias delimitan reglas para cubrir las necesidades de todos sus integrantes y establecen un sistema de comunicación familiar, con acuerdos y compromisos.

La investigación ha demostrado que los estilos autoritario, permisivo y negligente están asociados con bajos niveles de rendimiento académico, así como con problemas emocionales y psicosociales de los hijos. Mientras que un estilo de crianza democrático puede tener excelentes resultados formativos en los niños porque los prepara para dialogar, tener conciencia sobre ellos mismos y los demás, y para desarrollar conductas prosociales. Estas últimas son definidas como las acciones que tienden a beneficiar a otros, e incluyen conductas cooperativas y de ayuda, acompañadas de sentimientos de afecto y empatía (Mega y Liesa, 2017).

Otro agente de socialización lo constituyen los medios electrónicos (televisión, radio e Internet), que son fuentes de modelamiento de la conducta, del pensamiento y de las emociones.

Entre los contextos de gran influencia en el desarrollo infantil de los niños de preescolar se ubican los juegos. Bruner (2003) explica que el juego es útil para el desarrollo muscular y sensorial del niño, para ejercitar la toma de decisiones y solución de problemas, y para adquirir habilidades lingüísticas y cognoscitivas. La interacción del niño con sus pares, dentro de un contexto de juego, se traduce en el aprendizaje de reglas, cooperación y comportamientos sociales (Linaza, 2013).

Los niños preescolares inician la comprensión de sus propias emociones y las de otros, aspectos que sientan las bases para que autorregulen su comportamiento y eviten arranques de conducta emocional, lo que pueden lograr cuando aprenden a pensar en las repercusiones de sus acciones. Este desarrollo cognoscitivo y emocional se asocia con la aparición de conductas prosociales que, a su vez, repercuten en comentarios positivos por parte de las personas del entorno, que mejoran el autoconcepto del niño, propiciando sentimientos y emociones positivas. En suma, a través del aprendizaje socioemocional se crea y se refuerza un círculo virtuoso que promueve el equilibrio psicológico del niño.

Pero dicho círculo en lugar de virtuoso puede ser negativo. Esto se presenta cuando en lugar de recibir modelos prosociales, con retroalimentación, reforzamiento y valores positivos, el niño recibe modelos de agresividad o poca tolerancia, es sometido a castigos frecuentes o intensos, o vive en ambientes donde son escasas las interacciones sociales positivas. En tales situaciones el niño puede tener dificultades socioemocionales, al no desarrollar la capacidad de poner los problemas en una perspectiva de razonamiento lógico y de relaciones causa-efecto, lo que implica inhabilidad en la búsqueda de soluciones para resolver sentimientos, falta de razonamiento moral, arranques de ira, además de bajos niveles de autoestima y de habilidades para relacionarse con otros. 
En suma, las situaciones familiares y ambientales adversas constituyen factores de riesgo -que se definen como aquellos que conllevan el peligro de que ocurra algo que atente contra la integridad física o psicológica de una persona. Mientras que las situaciones que promueven el desarrollo socioemocional del niño pueden ubicarse como factores de protección -aquellos que contribuyen a disminuir la probabilidad de que tenga lugar un desajuste personal.

\section{Revisión de investigaciones empíricas sobre habilidades sociales y cognitivo-emocionales en el contexto hispano y latinoamericano}

Los estudios que se presentan a continuación fueron realizados con poblaciones adolescentes e infantiles, por lo que sus hallazgos son importantes para los objetivos del presente escrito.

\subsection{Estudios enfocados a la evaluación}

Se han desarrollado estudios, con amplias muestras de adolescentes, que aportan datos sobre la estrecha relación entre habilidades socioemocionales y rendimiento académico: en México (González et al., 2018; Guevara et al., 2017), en España (Gutiérrez y Expósito, 2015; Santamaría y Valdés, 2017), en Argentina (Coronel et al., 2011; Malander, 2016), y en Brasil (Amaral et al. 2015). Uno de los hallazgos consistentes es que los jóvenes con problemas emocionales muestran también problemas de índole social y académica, mientras que aquellos con alta habilidad social tienen mejor autoconcepto, más autoverbalizaciones positivas, menores niveles de ansiedad y mejor rendimiento escolar.

El estudio de Coronel et al. (2011) indicó que los jóvenes de nivel socioeconómico bajo presentaron aislamiento social y comportamientos que pueden constituirse en factores de riesgo; mientras que los de nivel socioeconómico alto exhibieron dimensiones facilitadoras de la socialización, como asertividad. Por su parte, Malander (2016) reporta que las prácticas parentales democráticas facilitaron que los niños y jóvenes desarrollaran estrategias y hábitos de estudio, motivación, manejo de ansiedad, concentración y dedicar tiempo a la preparación de exámenes; lo cual impacta en el rendimiento académico.

La investigación de Barrios y Frías (2016) corroboró que entre mayor sea el apoyo que brindan los padres y la escuela, su relación con los jóvenes será más positiva y comunicativa, lo que se relaciona con mejores habilidades socioemocionales; mientras Amaral et al. (2015) señalan que la carencia de habilidades sociales se asocia con comportamiento agresivo y delincuencia, relaciones conflictivas y bajo nivel educativo.

Las investigaciones realizadas con niños reportan resultados similares. El estudio de Isaza y Henao (2011), con niños colombianos de 2 y 3 años de edad, indicó que en las familias con tendencia democrática los niños mostraron un repertorio amplio de habilidades sociales, y ocurrió lo contrario en las familias con una estructura autoritaria o con poco manejo de normas.

En la investigación de Acevedo y González (2015) participaron 481 niños con edades entre 10 y 12 años, de 58 escuelas primarias de una ciudad mexicana; el estudio tuvo como objetivo identificar y describir las prácticas violentas entre los alumnos. Se diseñó la Cédula de Percepciones de las Dinámicas Escolares (78 ítems con una consistencia interna adecuada). Se identificaron en los alumnos diversos tipos de violencia, desde faltas de respeto, mentiras y manipulación, hasta actitudes que pueden tener consecuencias psicológicas, como: hacer comentarios negativos sobre otros, molestar a los demás por sus defectos, insultar, burlarse, rechazar, expresarse agresivamente y obligar a otros a realizar sus tareas.

Carbonero et al. (2015) evaluaron a 235 alumnos españoles de tercer ciclo de Educación Primaria con la Escala de Evaluación de las Actitudes de Responsabilidad Social del Alumnado de Educación Primaria y la Escala de Evaluación de Actitudes Generales hacia el Estudio (con valores aceptables de consistencia interna). Se encontraron diferencias significativas en las actitudes hacia el estudio y en la responsabilidad de los alumnos, en función de su rendimiento académico.

Los estudios hasta aquí descritos utilizaron cuestionarios, entrevistas y escalas de auto-reporte para evaluar las variables socioemocionales (instrumentos más comunes en las investigaciones de campo); esta 
característica hace que sean escasos los estudios que se realicen con niños preescolares.

Lacunza et al. (2009) construyeron y validaron tres Escalas de Habilidades Sociales para niños de 3, 4 y 5 años de edad, aplicándolas a 318 padres de niños argentinos en situación de pobreza. El instrumento para cada edad cuenta con 12 ítems y su consistencia interna fue adecuada; evalúa habilidades de interacción, resolución de conflictos, emociones y normas sociales, cooperación y expresión de sentimientos.

Un reporte posterior (Lacunza y Contini, 2009) presenta los resultados de la aplicación de tales instrumentos, indicando que los niños mostraron comportamientos sociales necesarios para su vida diaria y que las relaciones que establecían pueden considerarse como un factor protector de su salud, porque contribuyen a su funcionamiento adaptativo. Pero estos resultados y conclusiones se basaron únicamente en las respuestas que los padres dieron respecto al comportamiento de sus hijos, lo que lleva a plantear la necesidad de contar con datos provenientes de otras fuentes, incluyendo la observación directa en diversos contextos situacionales.

Zych et al. (2016) observaron a 38 niños españoles en cuatro situaciones diferentes: durante la clase, al realizar alguna tarea, a la hora de la salida y durante actividades de juego. Además de la observación directa utilizaron tres escalas respondidas por los docentes. Los autores corroboraron la relación entre afecto, ajuste escolar y desempeño académico, y que los alumnos que expresan emociones negativas durante el juego desarrollan bajos niveles de competencia social.

En otro estudio con niños preescolares españoles, Cepa et al. (2016) desarrollaron y aplicaron la Escala Reconocimiento, Regulación, Empatía y Resolución de Problemas (65 ítems con una consistencia interna aceptable). Los participantes obtuvieron puntuaciones altas en conciencia emocional y regulación de emociones, pero no en empatía y resolución de problemas. Los autores concluyen que en la sociedad actual, en la que las nuevas tecnologías favorecen poco la competencia emocional, es importante considerar a la escuela como la institución para promoverla.

\subsection{Estudios enfocados a la intervención}

Entre los autores pioneros de habla hispana dedicados al diseño de programas para promover la competencia social destaca Monjas (2002), quien aplica los principios de la psicología cognitivo-conductual para desarrollar una propuesta dirigida a profesores y padres de familia de niños inscritos en Educación Infantil (preescolar), Primaria y Secundaria. Monjas explica que esta perspectiva psicológica asume un Modelo de Competencia, considera al individuo desde un enfoque pedagógico y educativo, y tiene como objetivo la enseñanza activa y deliberada de comportamientos adaptativos a través de cuatro elementos: 1) enseñanza de habilidades específicas que se practican y se integran al repertorio conductual del individuo, a través de técnicas conductuales; 2) reducción de la ansiedad en diversas situaciones, promoviendo la relajación y conductas alternativas; 3) reestructuración cognitiva, incluyendo pensamientos, creencias y lenguaje autodirigido, para favorecer autoestima, y 4) solución de problemas y autorregulación.

El programa de Monjas (2002) está dirigido a la enseñanza activa y directa de seis áreas de comportamiento: de interacción social, para hacer amigos, conversacionales, relacionadas con sentimientos, emociones y opiniones, para resolver problemas interpersonales, y para relacionarse con adultos. Incluye diversas actividades ad hoc, divididas por grados de dificultad, para promover que los alumnos ubiquen la importancia de cada habilidad y la desarrollen, para lo cual se utilizan técnicas de modelamiento, ejemplificación, juegos de roles, retroalimentación, reforzamiento, práctica y ensayo conductual, asignando tareas para promover la transferencia de habilidades.

A partir de programas como el descrito, diversos estudios con niños y adolescentes han probado la efectividad de las técnicas cognitivo-conductuales para desarrollar habilidades sociales dentro del contexto escolar. Tales intervenciones se han realizado en España, con alumnos de nivel secundaria (Morales et al., 2018) y en escuelas públicas rurales (Cejudo, 2017); en Bolivia (Arancibia y Péres, 2007), y en México, con alumnos institucionalizados (González et al., 2012), con estudiantes de zonas rurales (Morales et al., 2013), 
y con alumnos que presentaban comportamiento disruptivo dentro del aula (García y Méndez, 2017). Todos los autores enfatizan la necesidad de desarrollar este tipo de programas en las escuelas para que los alumnos interactúen positivamente, adquieran autoestima, automotivación, y aprendan a regular sus emociones.

Otros programas se implementan a través de actividades de juego, como el realizado en México por Osés et al. (2016), cuya investigación cuasiexperimental reportó los efectos positivos de un programa de intervención en juegos cooperativos y creativos sobre el comportamiento asertivo y las estrategias cognitivas de resolución de situaciones sociales.

Justicia et al. (2006) exponen que los programas dirigidos a desarrollar habilidades socioemocionales deben: 1) iniciar tempranamente, desde la educación Inicial, 2) estar dirigidos a la prevención del desarrollo de conductas violentas, 3) contar con la colaboración de todos los agentes e instituciones de la comunidad, incluyendo padres, profesores, directivos y profesionales de la psicopedagogía, y 4) contar con planes sistemáticos, porque los programas eficaces incluyen orientaciones y ejercicios de práctica de habilidades concretas.

Entre los escasos programas de intervención dirigidos a niños en edad preescolar sobresale el implementado por de Miguel (2014), quien utilizó el programa de Monjas (2002) para desarrollar habilidades de interacción social en un grupo de niños españoles en riesgo de exclusión social, residentes en un centro de acogida. Se utilizó el Código de Observación de la Interacción Social (de Monjas et al., 1991, como se citó en de Miguel, 2004), que permitió observar interacciones en situaciones naturales y no estructuradas. Este sistema es considerado el método imprescindible y prototípico en evaluación conductual del proceso de enseñanza-aprendizaje. Los participantes mejoraron sustancialmente en todas las habilidades de interacción social.

Justicia et al. (2008) diseñaron un programa para el desarrollo de la competencia social y prevención del comportamiento antisocial, denominado Aprender a convivir. En el estudio realizado para probar su efectividad participaron alumnos españoles de educación Inicial. El programa tuvo una duración de 12 sesiones en igual número de semanas y se dividió en cuatro bloques: Normas de convivencia y su cumplimiento en el contexto escolar, Sentimientos y emociones, Habilidades de comunicación, y Ayuda y cooperación.

La investigación fue cuasiexperimental, pretest-postest con grupo experimental y de control. Se utilizó la Escala de Observación del Comportamiento Infantil en su versión para profesorado y la Escala de Observación en Educación Infantil-Revisada. Todas las subescalas utilizadas obtuvieron índices de ajuste adecuados. El análisis de datos indicó avances significativos entre los beneficiarios del programa en las áreas de la competencia social, y una disminución de las puntuaciones relativas al comportamiento antisocial, con diferencias significativas respecto al grupo control.

Fernández-Cabezas et al. (2011) presentaron nuevas evidencias del éxito del programa. Alba et al. (2013) señalan que el programa Aprender a convivir es una propuesta educativa implementada en diferentes ciudades españolas (Granada, Zaragoza) y en otros países de habla hispana (Bolivia, República Dominicana), que ha obtenido resultados significativos para el aprendizaje de la competencia social y la reducción de problemas de conducta. La principal aportación de este programa es que retoma las características del programa de Monjas (2002) y lo enriquece con recursos como marionetas, cuentos, juegos, canciones, murales, cómics y otros materiales específicos para cada una de las actividades. La investigación de JusticiaArráez et al. (2014) se encaminó a diseñar el sistema de Registro de Observación para el Programa Aprender a convivir y realizar el análisis de su estructura factorial y consistencia interna. Los autores muestran los resultados de los análisis exploratorios y confirmatorios, reportando índices de fiabilidad adecuados.

Por su parte, Benavides et al. (2016) realizaron un estudio para conocer el grado de satisfacción de las familias participantes en el programa piloto Aprender a convivir en casa. Los resultados indicaron que los participantes coinciden en la importancia de poner en práctica programas dirigidos a los padres de familia desde el ámbito escolar, manifestando opiniones positivas y satisfacción con el programa. 
Un programa con niños mexicanos preescolares fue implementado por Márquez et al. (2016), enfocado a desarrollar habilidades de lenguaje oral para solucionar conflictos. El diseño pretest-postest, a través de registros anecdóticos, bitácora de seguimiento y lista de cotejo, comprobó que el programa promovió en los niños el uso del lenguaje oral encaminado a la solución de conflictos con sus compañeros y con algunos familiares.

La Secretaría de Educación Pública de México (SEP) desarrolló el Programa Nacional de Convivencia Escolar Aprendamos a Convivir, cuyo objetivo general es favorecer el establecimiento de ambientes de convivencia escolar armónica, pacífica e incluyente, que coadyuven a prevenir situaciones de acoso escolar en escuelas públicas de educación básica, propiciando condiciones para mejorar el aprovechamiento escolar. En 2017 dio inicio su implementación en los seis niveles de Primaria, y en 2018 en Preescolar y Secundaria. Se diseñaron los Cuadernos de actividades para el alumno y la Guía para el maestro, que contienen dibujos y actividades para abordar seis ejes temáticos: autoestima, reconocimiento y manejo de emociones, convivencia, reglas, manejo y resolución de conflictos, y todas las familias son importantes. La tabla I muestra una comparación de los programas citados, considerando aquellos diseñados para ser integrados como parte del currículum escolar.

Tabla I. Comparación de programas curriculares para el desarrollo de habilidades socioemocionales

\begin{tabular}{|c|c|c|c|}
\hline Características metodológicas y pedagógicas & $\begin{array}{l}\text { Monjas } \\
(2002)\end{array}$ & $\begin{array}{l}\text { Justicia et } \\
\text { al. (2008) }\end{array}$ & $\begin{array}{l}\text { SEP/México } \\
\quad(2016)\end{array}$ \\
\hline $\begin{array}{l}\text { Plantea objetivos claros por grados de dificultad } \\
\text { para la enseñanza de habilidades específicas }\end{array}$ & $\checkmark$ & $\checkmark$ & $\checkmark$ \\
\hline Modelamiento por parte del profesor y compañeros & $\checkmark$ & $\checkmark$ & $\checkmark$ \\
\hline Instrucciones específicas del docente & $\checkmark$ & $\checkmark$ & \\
\hline Ensayo conductual de habilidades concretas & $\checkmark$ & $\checkmark$ & $x$ \\
\hline $\begin{array}{l}\text { Ejemplos de comportamientos adecuados } \\
\text { generados por los niños }\end{array}$ & $\checkmark$ & $\checkmark$ & $x$ \\
\hline $\begin{array}{l}\text { Juego de roles para aprender y practicar conductas } \\
\text { específicas en situaciones concretas }\end{array}$ & $\checkmark$ & $\checkmark$ & $x$ \\
\hline Práctica positiva & $\checkmark$ & $\checkmark$ & $x$ \\
\hline Retroalimentación por parte del profesor & $\checkmark$ & $\checkmark$ & $\begin{array}{c}\text { Sólo durante } \\
\text { la discusión } \\
\text { grupal }\end{array}$ \\
\hline Reforzamiento explícito & $\checkmark$ & $\checkmark$ & $x$ \\
\hline $\begin{array}{l}\text { Reducción de ansiedad. Relajación y conductas } \\
\text { alternativas }\end{array}$ & $\checkmark$ & $\checkmark$ & $x$ \\
\hline $\begin{array}{l}\text { Reestructuración cognitiva y su práctica, incluyendo } \\
\text { pensamientos, creencias y lenguaje autodirigido }\end{array}$ & $\checkmark$ & $\checkmark$ & $x$ \\
\hline $\begin{array}{l}\text { Habilidades programadas: interacción social, para } \\
\text { hacer amigos, conversacionales, relacionadas con } \\
\text { sentimientos, emociones, opiniones y conductas, } \\
\text { resolver problemas interpersonales, y relacionarse } \\
\text { con adultos }\end{array}$ & $\checkmark$ & $\checkmark$ & Parcialmente \\
\hline $\begin{array}{l}\text { Se enfoca a la solución de problemas y la } \\
\text { autorregulación }\end{array}$ & $\checkmark$ & $\checkmark$ & $x$ \\
\hline $\begin{array}{l}\text { Asignación de tareas para promover transferencia, } \\
\text { involucrando a los padres }\end{array}$ & $\checkmark$ & $\checkmark$ & \\
\hline $\begin{array}{l}\text { Materiales y actividades lúdicas que favorecen la } \\
\text { motivación y el involucramiento: marionetas, } \\
\text { cuentos, juegos, canciones, murales }\end{array}$ & Parcialmente & $\checkmark$ & Parcialmente \\
\hline $\begin{array}{l}\text { Evaluación: } 1 \text {. Se aplican instrumentos validados } \\
\text { antes y después de la intervención. } 2 \text {. Se realizan } \\
\text { registros conductuales durante la intervención. 3. Se } \\
\text { utilizan los datos para conocer los avances y verificar } \\
\text { el cumplimiento de objetivos }\end{array}$ & $\checkmark$ & $\checkmark$ & $x$ \\
\hline
\end{tabular}




\section{Discusión y conclusiones}

Como se desprende de lo expuesto, la competencia social incluye un conjunto de habilidades conductuales, pensamientos y emociones que permiten a los individuos relaciones interpersonales satisfactorias, sentimientos de bienestar en relación con los demás, logro de sus objetivos y una comunicación eficaz. Desafortunadamente no todos los hogares, comunidades y centros escolares han considerado la importancia de promover la competencia socioemocional de los niños de una manera clara y explícita.

Dentro de las escuelas preescolares se deben implementar programas educativos, con un enfoque preventivo, que definan objetivos claros y que contengan actividades y procedimientos sistemáticos encaminados a la enseñanza-aprendizaje de la competencia social.

El primer componente de un programa educativo es la definición y secuenciación de objetivos, considerando grados de complejidad, porque la investigación psicológica ha demostrado que las conductas socioemocionales que llevan a la competencia social se desarrollan de lo básico a lo complejo. Tales objetivos deben enunciarse con claridad, haciendo referencia a conductas concretas que sean observables, solo así el docente sabrá si sus objetivos educativos se han alcanzado. Los programas analizados cumplen con estas características, lo que constituye un acierto.

También puede considerarse que existe coincidencia entre los programas revisados, en cuanto a las áreas socioemocionales que sus objetivos abarcan: desarrollar estrategias para incrementar autoestima y autoconcepto, reconocimiento de emociones propias y de los demás, entablar diálogos para solucionar conflictos y conseguir un bien común, trabajar cooperativamente en actividades prosociales y proambientales, controlar emociones y pensamientos negativos, ayudar a otros a experimentar emociones positivas y reducir las negativas, mostrar aceptación hacia las demás personas, así como cortesía y amabilidad, y analizar problemas para lograr su solución.

Sin embargo, existen diferencias importantes en la metodología a seguir para el logro de tales objetivos, lo que guarda relación con el enfoque teórico-metodológico que se adopta. Desde el modelo cognitivoconductual, un programa escolar debe recurrir a estrategias multimodales de intervención, lo que implica utilizar un conjunto de técnicas; así se subsanan las debilidades que pueda tener el uso de técnicas separadas, aumentando el impacto, la durabilidad y la generalización de sus efectos (Caballo, 1993).

Lo anterior no es tomado en cuenta en programas como el de la SEP (2016), cuyas actividades se limitan a: promover la discusión y la reflexión entre los alumnos; dibujar e iluminar para ubicar ejemplos de modelos de ciertos comportamientos, y a plantear situaciones hipotéticas.

Como señalan Ríos y Marchena (2017), la enseñanza puede apoyarse en la instrucción verbal y el análisis de situaciones éticas y sociales, para favorecer el enfoque analítico de problemas y emociones, pero principalmente debe enfocarse a la práctica. Es decir, a ejercitar y practicar las habilidades, y convertirlas en respuestas adaptativas integradas al repertorio conductual de la persona.

A través de múltiples evidencias científicas de su efectividad, la psicología cognitivo-conductual ha aportado una estrategia multimodal que comprende un variado grupo de técnicas o procedimientos de enseñanza, que está compuesto por: instrucción verbal, diálogo y discusión, ubicación de ejemplos, modelamiento, práctica y ensayo conductual, retroalimentación (feedback), reforzamiento de comportamientos adecuados, juegos de roles, y tareas para casa (de Miguel, 2014; Márquez et al., 2016; Monjas, 2002; Osés et al., 2016).

Además de los objetivos secuenciados, los procedimientos de enseñanza directa y el análisis dialogado de situaciones pertinentes, un programa debe considerar el diseño de actividades y materiales adecuados al grupo de estudiantes. Los programas educativos preescolares deben aprovechar las múltiples oportunidades de enseñar a los niños habilidades sociales y emocionales. Sin dejar de lado la enseñanza informal que los profesores pueden proveer durante actividades de dibujo, juego locomotor o juegos tradicionales, pueden ubicarse tres tipos de actividades que son un marco ideal para implementar un 
currículum formal de enseñanza socioemocional en preescolar: juego cooperativo, juego de roles y lectura dialogada de cuentos.

Durante el juego cooperativo los niños realizan juntos una actividad, como elaborar o construir algo; tales situaciones cooperativas promueven una comunicación abierta, intereses comunes, confianza mutua y asumir la responsabilidad del propio aprendizaje. En el juego de roles (simulación social), cada niño puede representar un papel y practicar diálogos, soluciones a problemas y conflictos, así como diversas habilidades sociales. El papel del profesor durante tales actividades de juego incluye modelar esas habilidades socioemocionales, dar retroalimentación y reforzar explícitamente a los alumnos.

Tales consideraciones son retomadas por los programas cognitivo-conductuales, como los de Monjas (2002), lo que ha fundamentado gran parte de su éxito; en este último, la inclusión de marionetas, juegos, canciones, murales y cuentos ha representado un acierto adicional porque tales recursos favorecen la motivación y el involucramiento de los alumnos.

La lectura dialogada de cuentos abre a los profesores de preescolar la oportunidad de hablar con los niños de sus emociones y de proveer modelos de expresión y regulación emocional, ubicar situaciones éticas y no éticas, ubicar conflictos y enfocar el análisis de los problemas para buscar una solución adecuada (Guevara y Rugerio, 2017). Desafortunadamente, esta actividad no ha sido aprovechada en los programas curriculares.

El último componente de un programa de habilidades socioemocionales es el sistema de evaluación que ponga en práctica. La evaluación debe realizarse, de manera continua, a través de todo el proceso de enseñanza-aprendizaje. Al inicio es deseable que se utilicen diversos instrumentos, como entrevistas y cuestionarios dirigidos a padres y profesores, así como observaciones y análisis de la conducta infantil, para detectar situaciones sociales específicas en las que se presentan conductas socialmente adecuadas e inadecuadas, así como para evaluar las habilidades cognitivas, emocionales y conductuales con las que cuenta cada alumno. Esos mismos instrumentos han de utilizarse al final del curso, para dar cuenta de los avances de los niños y del grado de cumplimiento de los objetivos.

La evaluación durante el proceso del programa ha de incluir, principalmente, la observación de la conducta de los alumnos y su autoevaluación, lo que promueve su participación e involucramiento directo en el proceso de enseñanza-aprendizaje. Contar con instrumentos confiables durante los tres momentos de evaluación es de suma importancia, porque ello permite conocer el grado de cumplimiento de los objetivos del programa.

Los señalamientos expuestos a lo largo del escrito se basan en la evidencia de investigaciones realizadas en el contexto de países de habla hispana, por lo que pueden considerarse lineamientos para un currículum de habilidades socioemocionales en centros preescolares latinoamericanos. Tales programas han de desarrollarse con la colaboración de psicólogos y pedagogos, y su implementación implica la formación de profesores y padres.

\section{Referencias}

Acevedo, J. y González, J. (2015). Manifestaciones de la violencia perversa en escuelas primarias del noreste de México. Enseñanza e Investigación en Psicología, 20(3), 275-285.

Alba, G., Justicia-Arráez, A., Pichardo, M. M. y Justicia, J. F. (2013). Aprender a convivir. Un programa para la mejora de la competencia social del alumnado de educación infantil y primaria. Electronic Journal of Research in Educational Psychology, 11(31), 883-904. http://dx.doi.org/10.14204/ejrep.31.13105

Amaral, M. P., Maia-Pinto, F. J. y Medeiros, C. R. (2015). Las habilidades sociales y el comportamiento infractor en la adolescencia. Subjetividad y Procesos Cognitivos, 19(2), 17-38.

http://dspace.uces.edu.ar:8180/xmlui/handle/123456789/3452 
Arancibia, G. y Péres, X. (2007). Programa de autoeficacia en habilidades sociales para adolescentes. Ajayu, 5(2), 133-155.

Barrios, M. y Frías, M. (2016). Factores que influyen en el desarrollo y rendimiento escolar de los jóvenes de bachillerato, Revista Colombiana de Psicología, 25(1), 63-82. http://dx.doi.org/10.15446/rcp.v25n1.46921

Baumrind, D. (1966). Effects of authoritative parental control on child behavior, Child Development, 37(4), 887-907. https://arowe.pbworks.com/f/baumrind 1966 parenting.pdf

Benavides, A., Fernández-Cabezas, M. Romero, M. y Pichardo, M. (diciembre, 2016). Satisfacción de las familias participantes en el programa piloto Aprender a convivir en casa. Trabajo presentado en el XXIV Congreso Internacional de Psicología INFAD, Almería, España.

https://doi.org/10.13140/RG.2.2.10032.56324

Bruner, J. (2003). Juego, pensamiento y lenguaje. Infancia: educar de 0 a 6 años, 78, 4-10.

https://www.observatoriodelainfancia.es/oia/esp/documentos ficha.aspx?id=1742

Caballo, V. (1993). Manual de evaluación y entrenamiento de las habilidades sociales. Siglo XXI.

Caballo, V., Salazar, I. y Equipo de Investigación CISO-A España (2017). Desarrollo y validación de un nuevo instrumento para la evaluación de las habilidades sociales: el Cuestionario de habilidades sociales (CHASO) Behavioral Psychology / Psicología Conductual: Revista Internacional Clínica y de la Salud, 25(1), 5-24. https://www.researchgate.net/publication/317025754

Carbonero, M., Martín, L., Monsalvo, E. y Valdivieso, J. (2015). Rendimiento escolar y actitudes personales y de responsabilidad social en el alumnado preadolescente. Anales de Psicología, 31(3), 990-999.

https://doi.org/10.6018/analesps.31.3.181161

Cejudo, J. (2017). Efectos de un programa de mejora de la inteligencia emocional sobre el ajuste psicosocial y el rendimiento académico en educación primaria. Infancia y Aprendizaje, 40(3), 503-530.

https://doi.org/10.1080/02103702.2017.1341099

Cepa, A., Heras, D. y Lara, F. (2016). Desarrollo emocional: evaluación de las competencias emocionales en la infancia. International Journal of Developmental and Educational Psychology, 1(1), 75-81.

https://doi.org/10.17060/ijodaep.2016.n1.v1.212

Coronel, C., Levin, M. y Mejail, S. (2011). Las habilidades sociales en adolescentes tempranos de diferentes contextos socioeconómicos. Electronic Journal of Research in Educational Psychology, 9(23), 241-262.

http://dx.doi.org/10.25115/ejrep.v9i23.1436

de Miguel, P. (2014). Enseñanza de habilidades de interacción social en niños con riesgo de exclusión. Revista de Psicología Clínica con Niños y Adolescentes, 1(1), 17-26.

https://www.revistapcna.com/sites/default/files/14 02.pdf

Fernández-Cabezas, M., Benítez, L., Fernández, E., Justicia, F. y Justicia-Arráez, A. (2011). Desarrollo de la competencia social y prevención del comportamiento antisocial en niños de 3 años. Infancia y Aprendizaje, 34(3), 337-347. https://doi.org/10.1174/021037011797238603

García, A. y Méndez, C. (2017). El entrenamiento en habilidades sociales y su impacto en la convivencia escolar dentro de un grupo de primario. Revista de Estudios y Experiencias en Educación, 16(30), 151-164. https://dialnet.unirioja.es/servlet/articulo?codigo=5890352

González, C., Ampudia, A. y Guevara, Y. (2012). Programa de intervención para el desarrollo de habilidades sociales en niños institucionalizados. Acta Colombiana de Psicología, 15(2), 43-52. 
González, C., Guevara, Y., Jiménez, D. y Alcázar, R. (2018). Relación entre asertividad, rendimiento académico y ansiedad en una muestra de estudiantes mexicanos de secundaria. Acta Colombiana de Psicología, 21(1), 116-127. https://doi.org/10.14718/ACP.2018.21.1.6

Guevara, Y. y Rugerio, P. (2017). Interacciones profesor-alumnos durante lectura de cuentos en escuelas preescolares mexicanas. Revista Mexicana de Investigación Educativa, 22(74), 729-749.

http://www.scielo.org.mx/pdf/rmie/v22n74/1405-6666-rmie-22-74-00729.pdf

Guevara, Y., Cárdenas, K., Reyes, V. y González, C. (2017). Niveles de ansiedad y comprensión lectora, en estudiantes mexicanos de escuelas secundarias. Revista Electrónica de Psicología Iztacala, XX(3), 1057-1077. https://www.iztacala.unam.mx/carreras/psicologia/psiclin/vol20num3/Vol20No3Art13.pdf

Guevara, Y., Rugerio, P. y Corona, A. (2018). Relationship between maternal and infant behaviour during story time. Journal of Education and Human Development, 7(2), 100-112.

https://doi.org/10.15640/jehd.v7n2a13

Gutiérrez, M. y Expósito, J. (2015). Autoconcepto, dificultades interpersonales, habilidades sociales y conductas asertivas en adolescentes. Revista Española de Orientación y Psicopedagogía, 26(2), 42-58.

http://revistas.uned.es/index.php/reop/article/view/15215

Isaza, L. y Henao, G. (2011). Relaciones entre el clima social familiar y el desempeño en habilidades sociales en niños y niñas entre dos y tres años de edad. Acta Colombiana de Psicología, 14(1), 19-30.

https://actacolombianapsicologia.ucatolica.edu.co/article/view/355/360

Justicia, F., Benítez, J., Fernández, M., Fernández, E. y Pichardo, C. (2008). Aprender a convivir: programa de prevención do comportamento antisocial na educación infantil [Aprender a convivir: programa de prevención de conductas antisociales en la educación infantil]. Cadernos de Psicoloxía, 32, 37- 47.

Justicia, F., Benítez, J., Pichardo, C., Fernández, E., García, T. y Fernández, M. (2006). Aproximación a un nuevo modelo explicativo del comportamiento antisocial. Revista Electrónica de Investigación Psicoeducativa, 4(2), 131-150. http://www.investigacion-psicopedagogica.org/revista/articulos/9/espannol LArt 9 117.pdf

Justicia-Arráez, A., Alba, G., Romero, M. y Quesada, A. (2014). Análisis de la estructura factorial del registro de observación del programa aprender a convivir en 3 años (ROAC-3). International Journal of Developmental and Educational Psychology, 4(1), 557-568. https://doi.org/10.17060/ijodaep.2014.n1.v4.831

Lacunza, A. y Contini, N. (2009). Las habilidades sociales en niños preescolares en contextos de pobreza. Ciencias Psicológicas, 3(1), 57-66. https://doi.org/10.22235/cp.v3i1.137

Lacunza, A., Castro, A. y Contini, N. (2009). Habilidades sociales preescolares: una escala para niños de contextos de pobreza. Revista de Psicología, 27(1), 4-28. https://doi.org/10.18800/psico.200901.001

Linaza, J. (2013). El juego es un derecho y una necesidad de la infancia. Bordón. Revista de Pedagogía, 65(1), 103-118. https://recyt.fecyt.es/index.php/BORDON/article/view/brp.2013.65107

Malander, N. (2016). Percepción de prácticas parentales y estrategias de aprendizaje en estudiantes secundarios. Revista de Psicología, 25(1), 1-19. http://dx.doi.org/10.5354/0719-0581.2016.42098

Márquez, A., Vega, L. y Poncelis, F. (2016). Solución de conflictos a través de la expresión del lenguaje oral en niños preescolares. En J. L. Costa (Ed.) Psicología y educación: presente y futuro (pp. 398-406). Asociación Científica de Psicología y Educación. 
Mega, M. y Liesa, M. (2017). El juego cooperativo como método para favorecer la inclusión y el desarrollo de conductas prosociales. Infancia, Educación y Aprendizaje, 3(2), 649-654.

https://doi.org/10.22370/ieva.2017.3.2.795

Mendo, S., León, B., Felipe, E., Polo, M. y Palacios, V. (2016). Evaluación de las habilidades sociales de estudiantes de educación social. Revista de Psicodidáctica, 21(1), 139-156.

https://doi.org/10.1387/RevPsicodidact.14031

Monjas, I. (2002). Programa de enseñanza de las habilidades de interacción social (PEHIS) para niños y niñas en edad escolar. CEPE.

Morales, L., Ruíz, P. Merchán, A. y Guil, R. (2018). Programa emocional en alumnado de segundo de E.S.o. con déficit en sus logros académicos. International Journal of Developmental and Educational Psychology, 3(1), 99-107. https://doi.org/10.17060/ijodaep.2018.n1.v3.1227

Morales, M., Benítez, M. y Agustín, D. (2013). Habilidades para la vida (cognitivas y sociales) en adolescentes de una zona rural. Revista Electrónica de Investigación Educativa, 15(3), 98-113.

https://redie.uabc.mx/redie/article/view/564

Organización de las Naciones Unidas para la Educación, la Ciencia y la Cultura. (2015). Replantear la Educación: ¿hacia un bien común mundial? http://unesdoc.unesco.org/images/0023/002326/232697s.pdf

Organización para la Cooperación y el Desarrollo Económico (Octubre, 2015). 50. Foro Mundial sobre Estadísticas, Conocimiento y Políticas. Transformando las políticas, cambiando vidas, Guadalajara, México. https://www.oecd.org/statistics/5WF\%20Summary\%20Report\%20Espagnol.pdf

Osés, M., Duarte, E. y Pinto, L. (2016). Juegos cooperativos: efectos en el comportamiento asertivo en niños de 6o. grado de escuelas públicas. Revista Electrónica de Investigación Educativa, 18(3), 176-186.

https://redie.uabc.mx/redie/article/view/886

Piaget, J., Lorenz, K. y Erikson, E. (1982). Juego y desarrollo. Crítica.

Pinto, N., Girón, G. y Mac-Lellan, W. (2015). Valores de la familia en la educación inicial. Revista Multidisciplinaria Dialógica, 12(1), 4-27. https://dialnet.unirioja.es/descarga/articulo/5237738.pdf

Ríos, L. y Marchena, E. (2017). Propuesta de un programa e-learning para desarrollar habilidades sociales. Enseñanza e Investigación en Psicología, 22(3), 301-311.

Santamaría, B. y Valdés, M. (2017). Rendimiento del alumnado de educación secundaria obligatoria: influencia de las habilidades sociales y la inteligencia emocional. International Journal of Developmental and Educational Psychology, 2(1), 57-66. https://doi.org/10.17060/ijodaep.2017.n1.v2.918

Secretaría de Educación Pública (2016). Programa Nacional de Convivencia Escolar. Secretaría de Educación Pública. http://convivenciaescolar.seph.gob.mx/ponencias/Bibliotecas\%20Escolares\%20PNCE.pdf

Weissberg, R., Durlak, J., Domitrovich, C. y Gullotta, T. (2015). Social and emotional learning. Past, present, and future. En J. Durlak, C. Domitrovich, R. Weissberg, y T. Gullotta, Handbook of social and emotional learning. Research and practice (pp. 3-19). The Guilford Press.

Zych, I., Ortega-Ruiz, R. y Sibaja, S. (2016). El Juego Infantil y el desarrollo afectivo: afecto, ajuste escolar y aprendizaje en la etapa preescolar. Infancia y Aprendizaje, 39(2), 380-400.

https://doi.org/10.1080/02103702.2016.1138718 